\title{
ESTRATEGIAS PARA LA RECUPERACIÓN DE PODER SINDICAL EN ESPAÑA*
}

\author{
José Pablo Calleja Jiménez \\ Dpto. Sociología. Universidad de Oviedo \\ Miembro del grupo de investigación PROMEBI
}

Orcid: 0000-0002-5391-5189

Recibido el 30 de septiembre de 2016

DOI: $10.1387 /$ lan-harremanak. 17498

Aceptado el 13 de octubre de 2016

\section{ABSTRACT}

Resumen: La crisis de los sindicatos tiene una dimensión mundial. Sin embargo, la gravedad del declive, los factores del debilitamiento y las estrategias disponibles para la recuperación dependen de cada sistema de relaciones laborales. Este texto comienza con un breve repaso de las aportaciones teóricas más relevantes acerca de las fuentes del poder sindical. Acudir a este nivel de análisis se considera el más operativo para la revitalización sindical, pues ubica las causas del debilitamiento y las estrategias para el fortalecimiento en las propias organizaciones sindicales. A continuación, el artículo realizará un recorrido por el estado de cada una de las fuentes de poder sindical en las principales organizaciones españolas a partir de entrevistas semi-estructuradas a sindicalistas e informantes-clave y concluirá con propuestas estratégicas derivadas del enfoque propuesto.

Palabras clave: sindicatos, revitalización, relaciones laborales

* Comunicación con la que participó en el II Encuentro de Profesionales del Asesoramiento Laboral y Social, celebrado en la Facultad de Relaciones Laborales y Trabajo Social (UPV/EHU-Leioa) el día 30 de septiembre de 2016. 
Abstract: Trade union crisis has a global dimension. However, the extent of the decline, weakening factors and available recovery strategies are depending on the labor relations system. This paper begins with a brief review of the principal theoretical contributions on union power sources. This level of analysis is considered the most operative for trade union revitalization because both weakening causes and strenghtening strategies are placed inside trade union organizations. The article then performs a report about the condition of each union power sources in the main trade union confederations. This report was made using several semi-structured interviews as a part of a more broad study. This paper will conclude with strategic proposals related to the suggested framework.

Keywords: trade unions, revitalization, labor relations 


\section{SUMARIO}

Sumario: 1. Introducción. 2. Las fuentes de poder sindical. 3. Metodología. 4. Resultados. 4.1. Poder asociativo. 4.2 Poder estructural. 4.3 Poder institucional. 4.4 Poder de coalición-alianzas. 4.5 Poder organizativo. 4.6 Poder estratégico. 4.7 Poder moral-discursivo. 5. Conclusiones. 6. Bibliografía.

\section{Introducción}

Los sindicatos iniciaron un proceso de declive a nivel mundial a partir de la década de 1980. Esta crisis sindical implica que han perdido afiliación y también influencia social, económica y política (Behrens et al., 2004a). Su origen se debe a una serie de transformaciones en los ámbitos laboral, económico y social que plantean retos a las organizaciones sindicales para los que no se están dando respuestas adecuadas. Según algunos autores (Ebbinghaus, 2002; Köhler, 2008) los sindicatos son actualmente unos «dinosaurios» de la era industrial, pero también de la economía de servicios, unas burocracias arcaicas que sobreviven en un medio cada vez más extraño a sus valores, formas de proceder y cultura. Esta situación hace que se consolide cada vez más una idea en el debate social y académico sobre el futuro de los sindicatos. Este devenir a largo plazo será la inevitable desaparición de las organizaciones sindicales o, todo lo más, su transformación en sindicatos corporativos en sectores como la administración pública, donde se cuenta con empleo estable y homogéneo.

Unos años más tarde del inicio del declive sindical mundial, comienza a consolidarse la corriente académica de Labor Revitalization Studies. Esta corriente comienza en el ámbito anglosajón, principalmente Estados Unidos, donde los sindicatos habían sido reducidos a mínimos hacia finales del siglo xx. Algunas experiencias exitosas en sectores muy precarios animan el interés académico por las transformaciones en las organizaciones sindicales para recuperar su papel como actores sociales destacados. Posteriormente, esta corriente de estudios se extiende por el viejo continente con contribuciones de países europeos, principalmente Reino Unido, Francia, Alemania. En esta corriente el caso español ha sido poco estudiado o se ha hecho de forma parcial y principalmente por autores foráneos (Hamann y Martínez-Lucio, 2003; Frege y Kelly, 2004).

El progreso social debe una parte importante a las organizaciones sindicales, por lo que su debilitamiento debería causar honda preocupación entre otros 
actores sociales. Los sindicatos contribuyen a la representación de intereses en las sociedades plurales, especialmente de los más desfavorecidos (Behrens et al., 2004a). No menos importante es la función que realizan para la redistribución de la riqueza, sin ser un mecanismo del Estado. Esta función, que los sindicatos realizan de forma casi exclusiva, es cada vez más necesaria ahora que los Estados se ven crecientemente presionados por los principios neoliberales que gobiernan la economía mundial. La competitividad es ahora el único argumento para poder tener presencia en los mercados globales y los gobiernos que regulan en contra de los intereses del capital son pronto reprimidos por medio de la financiación, la inversión foránea u otros mecanismos. Ante esta situación, los sindicatos son mecanismos de redistribución y cohesión social cada vez más importantes, a la vez que son compatibles y hasta necesarios para alcanzar los objetivos de competitividad. La importancia y trascendencia de las organizaciones sindicales requiere aunar esfuerzos para su revitalización en estos tiempos de crisis. Las aportaciones de la corriente de Labor Revitalization Studies buscan contribuir a dar soluciones prácticas a las organizaciones sindicales para basar estrategias de empoderamiento.

Para explicar el debilitamiento del poder sindical podemos hacer referencia a factores externos como la globalización, el neoliberalismo, la crisis ideológica e identitaria de la izquierda social, la falta de alternativas al capitalismo, los nuevos modos de gestión de recursos humanos, los cambios en el sistema productivo, etc. Estos factores son reconocibles pero la posibilidad de modificarlos tiende a escaparse de las capacidades y del alcance del sindicalismo y sus organizaciones. Por tanto, lo que cabe es preguntarse por los factores internos del debilitamiento, aquellos sobre los que el sindicalismo sí que tiene capacidad y dominio. El punto de partida en este enfoque es que los sindicatos no han sabido adaptarse a las circunstancias cambiantes. Desde esta visión, que mira hacia dentro de las organizaciones, es desde donde se puede analizar la capacidad de los sindicatos para aumentar su poder a través de la renovación interna.

A continuación, utilizando las aportaciones teóricas de la corriente mencionada, conceptualizaremos y tipificaremos el poder sindical para, posteriormente, construir a este nivel el marco de análisis sobre los sindicatos españoles y su revitalización.

\section{Las fuentes de poder sindical}

El poder sindical ha de ser entendido como la capacidad de imponer las posiciones sindicales ante sus interlocutores. Siempre que se da una lucha de poder, cada parte evalúa previamente su capacidad para poder ganar y también el coste de participar en la lucha. En las últimas décadas, la debilidad del poder sindical hace que las organizaciones se caractericen por una posición defensiva 
y generalmente reactiva, lo que contribuye aún más a su declive. Esta situación hace que las cuestiones relativas al poder estén en el centro del debate sobre la revitalización sindical.

El poder sindical ha sido estudiado por muchos autores en las últimas décadas (Hyman, 1981; Batstone, 1988; Gall, 2008; Levésque y Murray, 2010; Kelly, 2011). En una aportación teórica reciente, Gumbrell-McCormick y Hyman (2013: 30-31) aún reconociendo la existencia de diferencias en la forma de ejercer el poder sindical en función del sistema de relaciones laborales, consideran que es posible identificar cuatro fuentes de poder sindical ampliamente reconocidas:

- Estructural: Derivada de la posición que ocupan los trabajadores que están organizados en un sindicato. Pueden ser portadores de habilidades escasas u ocupar una posición estratégica en la producción, lo que les concede poder de negociación. A mayor poder en la negociación, mayor fortaleza sindical.

- Asociativo: El simple hecho de tener miembros aporta recursos humanos y financieros a un sindicato. El poder asociativo refleja la afiliación, la «voluntad para pagar» de los miembros (Offe y Wiesenthal, 1985: 80).

— Organizacional: La «voluntad para actuar» de los afiliados (ibíd.) requiere un proceso de identificación con el sindicato y sus políticas. Generar recursos de poder asociativo es una precondición obvia para desarrollar poder organizacional que además requiere de procesos democráticos y de diálogo interno para diseñar políticas comprendidas, compartidas y elaboradas a todos los niveles de la organización.

-Institucional: La fortaleza en los ámbitos asociativo y organizacional ha de estar promovida a través de medidas legales, reconocimiento por la parte empresarial, participación en estructuras consultivas o a través de la administración de políticas sociales públicas. No obstante, este tipo de apoyos resulta precario a largo plazo si obtener poder institucional va en detrimento de las demás fuentes de poder sindical.

La actual situación de debilidad de los sindicatos revela la necesidad de recabar poder de fuentes supletorias que, sin ser necesariamente nuevas, pueden haber sido poco atendidas anteriormente. Gumbrell-McCormick y Hyman (2013: 30-31) las consideran como una vía importante para la recuperación del poder y la influencia sindical en momentos de declive:

- Moral (discursivo-comunicativo): Representar los ideales de justicia social, divulgar la misión y la visión de los sindicatos en su compromiso con la sociedad. Requiere de mecanismos democráticos para la elaboración y una comunicación actual y efectiva. 
- Colaboración (coalición): Establecer alianzas con otras organizaciones, grupos, movimientos ciudadanos, etc. Las características diferenciadoras de los sindicatos con respecto a otras entidades sociales hace que la búsqueda de sinergias sea una tarea difícil, pero no imposible.

- Estratégica (logistica): Utilización eficaz y eficiente de los escasos recursos disponibles, lo que requiere de conocimiento, planificación e innovación.

\section{Metodología}

La principal herramienta para la recogida de información fueron entrevistas semi-estructuradas a sindicalistas e informantes-clave. Se realizaron un total de 42 entrevistas a personas de los sindicatos considerados «más representativos» a nivel nacional y autonómico en los términos establecidos por la legislación vigente $^{1}$ y añadiendo el sindicato USO por su relevancia en la escena sindical española ${ }^{2}$.

En las entrevistas se trató que hubiera representación de todos los niveles organizativos de las organizaciones sindicales. A nivel territorial predomina el nivel de confederación (14 entrevistas), seguido del autonómico (11). Dentro de las federaciones, se hicieron 7 entrevistas a federaciones de ámbito autonómico y 5 de ámbito nacional, además de 5 entrevistas a expertos externos a los sindicatos (consultores y académicos). Las entrevistas contenían, entre otros aspectos, una batería de preguntas sobre las fuentes de poder sindical tradicionales o complementarias y su situación actual dentro de las organizaciones, siempre con miras a la revitalización sindical.

\section{Resultados}

La información obtenida tras la transcripción de las entrevistas y el análisis se ha categorizado y, posteriormente, se ha puesto en relación con la producción teórica de la corriente de Labor Revitazation Studies para ofrecer los siguientes resultados.

\subsection{Poder asociativo}

El desarrollo del poder asociativo siempre aumenta las posibilidades de revitalización sindical. Aumentar el número de afiliados permite desarrollar im-

\footnotetext{
${ }^{1}$ Ley Orgánica 11/1985, de 2 de agosto, de Libertad Sindical.

2 Entrevistas realizadas por organización del entrevistado: 14 de CCOO, 14 de UGT, 3 de USO, 3 de ELA, 2 de CIG, 1 de LAB y 5 externos.
} 
portantes sinergias que se extienden a otras fuentes de poder sindical. Pero no solamente se trata de la cantidad de afiliados, es necesario también atender a la calidad de la dimensión afiliativa, lo que significa su conexión con la realidad de la población asalariada y la integración y vitalidad dentro de la organización. Dentro del modelo de sindicatos generales que predomina en el sindicalismo español, es necesario promover la inclusión de todos los tipos de trabajadores y de todas las características socio-demográficas. Sin embargo, los sindicatos españoles tienen una afiliación cuya estructura no refleja la realidad del mercado laboral. La abundancia de determinados tipos de trabajadores y la ausencia de otros puede extender la segmentación laboral al terreno del sindicalismo, lo que conlleva el riesgo de corporativización y obstaculiza las posibilidades de revitalización. Además, los sindicatos españoles, que se caracterizan por una alta fluctuación en su afiliación — con una gran cantidad de altas pero también de bajas-y en los últimos años están comenzando a perder afiliados en su balance neto sin haber desarrollado estrategias eficaces para remediarlo. Entre las principales acciones para contrarrestar esta disminución de miembros se incluyen la adaptación de la legislación sindical a la realidad laboral, mejorando el modelo de representación y ser capaces de desarrollar incentivos selectivos positivos verdaderamente funcionales para captar y retener afiliados, habiendo fracasado el modelo de servicios no sindicales (seguros, viajes, descuentos). Otros retos de las organizaciones sindicales para el desarrollo del poder asociativo tienen relación con otras fuentes de poder sindical, tradicionales y complementarias. En este sentido, las cuestiones organizativas y los aspectos democráticos internos, el desarrollo y la práctica de valores morales, el establecimiento de alianzas y la eficacia de la acción sindical, influyen notablemente en el desarrollo del poder asociativo.

\subsection{Poder estructural}

El poder estructural de los sindicatos ha sido una de las mayores víctimas de los cambios recientes en el mundo económico y laboral. Procesos como la globalización o las transformaciones organizativas de las empresas, han disminuido la capacidad de los sindicatos para imponer sus demandas. Este poder tampoco es ajeno a cambios sociales y políticos derivados de la desideologización y la institucionalización, que han supuesto una menor capacidad sindical para la movilización. La pérdida de poder estructural es muy preocupante pues conlleva la pérdida de eficacia y utilidad de las organizaciones sindicales para los trabajadores, lo que se traslada a dimensiones como la afiliación o la influencia económica y política. Aumentar la posición y la capacidad negociadora de los sindicatos requiere, además del desarrollo de otras fuentes de poder sindical, ser capaz de innovar en las estrategias a desarrollar en la negociación colectiva. Los sindicatos españoles han demostrado esta capacidad en sectores altamente expuestos a la competitividad internacional. No obstante, en ocasiones lo han hecho por es- 
tar sometidos a fuertes presiones por la parte empresarial y deben tener cuidado de no perder la legitimidad ante los trabajadores. Fortalecer este poder requiere también la innovación en la acción colectiva que necesita de nuevas expresiones para las que son necesarias sinergias con otros actores y establecer alianzas.

La segmentación de la fuerza laboral española puede obstaculizar seriamente las capacidades para dar respuestas sindicales efectivas. Desde el punto de vista de la revitalización, sería importante que el sindicalismo español tuviera una seńa de identidad clara y coherente, pero existe una gran fragmentación, competitividad y extrańamiento entre las organizaciones sindicales. Esta situación conduce en la práctica a un particularismo sindical que entorpece las posibilidades de alcanzar el objetivo de revitalización del conjunto del movimiento sindical. La práctica habitual de todas las organizaciones sindicales es tratar de desplazar la negociación colectiva al ámbito en el que tienen más fuerza, rechazando los demás. Además, en la situación actual, los sindicatos estatales mayoritarios se ubican en un papel institucional desde el que tratan de desarrollar estrategias contradictorias que oscilan entre la movilización y el diálogo social, instrumentos que se han debilitado mucho en los últimos años. El peso de estas grandes organizaciones en el sindicalismo español hace que sus errores estratégicos tengan un impacto significativo en el debilitamiento de la posición sindical para el conjunto del Estado.

\subsection{Poder institucional}

El poder institucional de los sindicatos es la existencia de mecanismos que impulsan la posición sindical en el sistema político. Lo deseable es que se produzca como resultado de la acumulación de otras fuentes de poder sindical y que este poder se utilice para mantenerlas o mejorarlas. Sin embargo, suele llevar a una situación paradójica y es que su obtención contribuye a descuidar otras fuentes de poder sindical o incluso lleva a sustituirlas por la complacencia que el poder institucional suele producir en las organizaciones sindicales. Así, si hay armonía con los poderes públicos o éstos quieren instrumentalizar a los sindicatos, pueden otorgarles poder institucional. Para las organizaciones es más difícil y trabajoso obtener poder sindical a través de otras fuentes independientes como la afiliación, la movilización, las alianzas o el eco en la sociedad, y pueden preferir el poder institucional que suele estar mejor dotado económicamente. Además, el excesivo peso de algunas estructuras sindicales españolas tiene un alto coste, lo que se traduce en una dependencia económica que puede menoscabar la capacidad de influencia política.

El poder institucional de los sindicatos españoles es claramente superior al resto de las fuentes del poder sindical y en esta diferencia reside su principal debilidad. Al contrario de otras fuentes de poder sindical no es un poder autónomo, sino dependiente. La falta de complementariedad con otras fuentes de 
poder por aspectos estructurales, organizativos y afiliativos hace que el poder institucional, que sólo proviene de un agente externo, sea graciable y, en consecuencia, débil. Los sindicatos españoles que participan en el diálogo social, han de analizar su estrategia en este ámbito con vistas a la revitalización sindical. Para ello, deberían tener en cuenta el amplio rechazo social que el actual «corporatismo de crisis» (Urban, 2012: 229) provoca y que se extiende a los sindicatos participantes. No obstante, rechazar totalmente el poder institucional podría ser una grave error puesto que bien utilizado sirve para impulsar y mantener medidas que mejoren la posición sindical (Baccaro et al., 2003). Por tanto, la participación institucional es causa del debilitamiento y una precondición para el fortalecimiento, donde el resultado dependerá en última instancia de cómo se utilice.

Las alianzas con movimientos sociales y ciudadanos, el desarrollo de acciones comunicativas eficientes, nuevas formas de acción colectiva y la acción sindical internacional, son formas innovadoras capaces de mejorar el papel político de los sindicatos. Obtener influencia política es determinante para el futuro del movimiento sindical espańol y si esa influencia se obtiene por medios autónomos será más efectiva y duradera. Sin perder de vista el marco del Estado-nación, al movimiento sindical europeo y mundial le urge ser capaz de obtener influencia política en las instancias supranacionales, un espacio en el que los intereses empresariales están muy asentados y desarrollan gran capacidad de interlocución y presión.

\subsection{Poder de coalición-alianzas}

Los sindicatos españoles pueden aumentar sus fuentes de poder sindical en todos los niveles organizativos mediante el establecimiento de alianzas. No obstante, desde el punto de vista de la elección estratégica, el liderazgo de los sindicatos mayoritarios no suele confiar en el establecimiento de alianzas con otros movimientos o grupos sociales y viceversa. La falta de confianza mutua es un obstáculo que, en función de las circunstancias, puede llegar a ser insalvable. Sin embargo, la debilidad de las organizaciones sindicales y la existencia actual de «oportunidades políticas» (Tarrow, 2004: 45) en forma de incentivos para la acción conjunta que se dan, sobretodo, por la existencia de una agenda común, puede favorecer el desarrollo de coaliciones.

Para que alcancen todo su potencial para la revitalización sindical los sindicatos deben optar por hacer coaliciones profundas, estables y duraderas, en lugar de puntuales alianzas superficiales de apoyo o, todo lo más, de apoyo mutuo (Tattersall, 2005), como las que habitualmente suelen desarrollar. Pero las coaliciones más efectivas requieren que las organizaciones sindicales lleven a cabo una profunda transformación que debe abarcar aspectos organizativos, comunicativos, culturales y estratégicos. La participación institucional de algunos sindicatos ma- 
yoritarios, en su rol de insider del sistema político, dificulta el establecimiento de alianzas con muchas organizaciones que desean ser outsider. Además, la incorporación de nuevos perfiles personales y profesionales, la extensión de procesos democráticos internos y la incorporación de activistas sociales en las estructuras sindicales, son algunos de los cambios organizativos necesarios para favorecer las alianzas.

La eclosión de nuevos movimientos sociales y expresiones de protesta popular de alto contenido político constituyen tanto una oportunidad como una amenaza para el movimiento sindical en su conjunto (Köhler y Calleja, 2015). Los sindicatos a través de las coaliciones profundas pueden acceder y ampliar recursos que ahora mismo están en un estado precario como son la movilización, la innovación en las acciones colectivas, la capacidad de influenciar el proceso político, adquirir una imagen más solidaria vinculada a intereses amplios de ciudadanía y mejorar los niveles de confianza entre la sociedad. La amenaza existe en la medida en que estas nuevas expresiones del conflicto social puedan desplazar o incluso marginalizar a los sindicatos, que ya no tienen el monopolio del conflicto social.

Por otro lado, algunos sindicatos minoritarios demuestran gran capacidad de formar «coaliciones de protesta» (McIlroy, 2000: 3) y junto con la importancia social que han adquirido algunos movimientos sociales recientes, pueden hacer que una alianza exitosa con algunas organizaciones sindicales sea poderosa. Esta situación puede llevar a la mayor fragmentación del movimiento sindical, lo que sería un obstáculo para su revitalización. Por otro lado, también podría llevar a la transformación del sindicalismo español, cuyo desarrollo es una incógnita.

\subsection{Poder organizativo}

Todos los sindicatos españoles han abordado restructuraciones dentro de sus organizaciones como reacción al debilitamiento de sus fuentes de poder sindical. En algunos casos, estos cambios han permitido mejorar la posición negociadora o la función institucional de los sindicatos. Las fusiones entre las federaciones, buscando crear economías de escala, ha sido una práctica bastante extendida. Las reestructuraciones internas, abordando cambios en el gobierno o en la administración sindical han sido menos frecuentes. En todos los casos, la motivación para llevarlas a cabo ha sido fundamentalmente defensiva y, en ausencia de una planificación estratégica para la revitalización sindical, muchas se quedan en meros «arreglos cosméticos» (Behrens et al., 2004b: 119) que aportan poco en términos de revitalización sindical.

Algunos de los retos que enfrenta el sindicalismo como la precariedad, el descenso de la afiliación y la representación de los trabajadores no se van resolver sólo con cambios organizativos, pero son el primer paso para enfrentarlos. Los sindicalistas y expertos entrevistados reclaman formulas organizativas más 
ágiles y flexibles. Destaca la apuesta por el nivel organizativo territorial a nivel local, el más cercano al trabajador y al ciudadano, como un nuevo ámbito para la acción y representación sindical. En este sentido, los pactos a nivel local entre sindicatos y administraciones públicas para garantizar un nivel de condiciones laborales en el territorio es una estrategia que ha resultado efectiva en el ámbito anglosajón (Bronfenbrenner et al., 1998). Otro de los asuntos destacados es la necesidad de mejorar la articulación entre los distintos niveles organizativos. Existen importantes riesgos por la concentración de poder y recursos en determinadas estructuras internas de las organizaciones sindicales. Generar más mecanismos de solidaridad interfederal o confederal podría ayudar a la acción sindical en aquellos sectores que más lo necesitan y que son los que disponen de menos recursos materiales y humanos. La necesidad de extender la participación y revisar los procesos de toma de decisiones interna, también es expresada y sentida. La mejora de la participación beneficia a la identidad colectiva y el compromiso con la organización, genera vitalidad interna y desarrolla el poder organizativo.

Por último, existen déficits en cuanto a la presencia de determinados perfiles en las estructuras sindicales. El más preocupante es el de los jóvenes, pues parece que no se garantiza el relevo de una generación que ahora mismo está copando los puestos en las organizaciones sindicales. Se produce demasiada rotación de las mismas personas por los cargos sindicales, lo que no deja espacios libres para nuevos activos. Las mujeres se han ido incorporando progresivamente a las estructuras sindicales, aunque deberían hacerlo en mayor medida y, sobre todo, en posiciones de mayor relevancia. Perviven ciertos rasgos machistas en los sindicatos que alejan a las muejeres de la participación, mientras que una política sindical sensible con la igualdad de género mejora siempre las posibilidades de revitalización. Otros colectivos como inmigrantes y trabajadores precarios o parados están muy ausentes de los puestos de las estructuras sindicales. La falta de representación de estos colectivos lastra la imagen, el discurso y la estrategia, con efectos negativos sobre el poder organizativo, que se extienden a otras fuentes de poder sindical.

\subsection{Poder estratégico}

Los recursos financieros de los sindicatos españoles son escasos y se están reduciendo cada vez más. Esta es una situación intolerable, dadas las funciones públicas que desarrollan en representación de todos los trabajadores. Además, la precariedad en el desarrollo de estas funciones va en contra de la eficacia y la utilidad, pudiendo contribuir aún más al declive sindical. Las opciones para remediar esta situación son diferentes y van desde la petición de una ley de financiación hasta la eliminación de la extensión de los efectos de la negociación colectiva. En cualquier caso, los sindicatos han de tratar de mejorar sus fuentes de financiación independientes. El papel de los sindicatos en la formación, merced 
a su participación institucional, o en otras políticas sociales, contribuye a aumentar el tamańo de las organizaciones con la creación de organismos parasindicales. Se hace necesaria una reflexión sobre los beneficios y los perjuicios que estas actividades comportan para el desarrollo de las fuentes de poder sindical. En tiempos de recursos escasos, es más necesario que nunca desarrollar poder estratégico, es decir, un uso racional y coherente de los recursos de la organización para desarrollar poder sindical, sin hacer dispendios ni derroches en acciones que no son verdaderamente útiles para los objetivos de los sindicatos.

\subsection{Poder moral-discursivo}

El poder moral y discursivo es una fuente de poder sindical complementaria cuyo desarrollo puede, al igual que las otras fuentes, generar efectos positivos que se extienden a otros aspectos de la acción sindical. Sin embargo, los sindicatos espańoles son una de las instituciones que inspiran más desconfianza en la sociedad, según los estudios del CIS (2015). Poner remedio a esta percepción social de los sindicatos es un asunto urgente, pues su continuidad afecta negativamente a las posibilidades que tienen los sindicatos de aumentar sus fuentes de poder y revitalizarse. Desarrollar poder moral no es tarea fácil, pues en la composición de los sindicatos no siempre se encuentran motivaciones solidarias, también hay quien se incorpora desde una visión utilitarista, egoísta y corporativista. A través de la vía dialógica (Offe y Wiesenthal, 1980), los sindicatos deben integrar las diferentes visiones e intereses para elaborar una identidad colectiva de base solidaria. Para ello, la extensión de la afiliación, el fomento de la participación, la ampliación de la representación en las estructuras y el estilo del liderazgo desarrollan un papel fundamental, creando las condiciones para el desarrollo del poder moral de los sindicatos.

Renovar el discurso sindical es necesario y ha de ser una estrategia planificada y adaptada a las diferentes circunstancias. Los entrevistados relatan situaciones en las que cambiar la imagen del sindicato y el mensaje ha funcionado y en otras que no, depende de las características de los trabajadores. Los sindicatos podrían utilizar sus propias encuestas de opinión y el desarrollo de otras técnicas de investigación para conocer los efectos de las comunicaciones que realizan, como hacen sus compañeros europeos (Kloosterboer, 2007). La comunicación hacia el exterior es fundamental y los sindicatos espańoles podrían mejorar sus perspectivas de revitalización a través de un uso eficaz de los medios de comunicación. Normalmente, la presencia de los sindicatos en los medios se produce de manera reactiva, mientras que una presencia más regular y proactiva en los medios, defendiendo los argumentos de las organizaciones sindicales y desplegando el poder moral del sindicato, contribuiría mejorar la percepción social. Renovar la imagen implica integrar a otros perfiles, contribuyendo así a mejorar la identificación con las organizaciones sindicales por parte de una fuerza asalariada que es cada vez más heterogénea. Sin embargo, el desarrollo de una estrategia comu- 
nicativa efectiva para el conjunto del sindicalismo se ve perjudicado por la división intersindical y por la falta de medios de comunicación realmente sensibles con los objetivos sindicales.

Un ámbito en el que se puede desarrollar poder moral a través de alianzas es el internacional. Existen movimientos internacionales en contra de la globalización, a favor de la lucha por los derechos humanos, la ecología, etc., con los que algunos sindicatos europeos están muy vinculados. Las oportunidades estructurales para la acción sindical que crea la globalización han de tener respuesta estratégica, utilizando la diversidad en forma de alianzas y mediante la concatenación de acciones globales y locales (Evans, 2010). Sumar coaliciones en el sindicalismo internacional multiplica el impacto de las acciones y puede contribuir al desarrollo de un ámbito de acción sindical que cada vez más necesario, incluso imprescindible para algunos de los entrevistados.

\section{Conclusiones}

La pérdida de influencia en los ámbitos social, político y económico junto con la disminución del número de afiliados caracteriza el declive de las organizaciones sindicales, que acumula ya cuatro décadas. Lastradas por la inercia de sus pesados aparatos, convulsas por los rápidos cambios en el mundo del trabajo y confundidas por la heterogeneidad creciente de una cada vez más desideologizada fuerza laboral, los sindicatos no están dando respuestas adecuadas a los retos que les plantea su entorno. Como los demás actores industriales, su ciclo de vida pasa por fases de creación, madurez y declive que conduce a la extinción o renovación. En la última fase, los cambios del entorno retan la validez y sostenibilidad del diseño organizacional anterior y la respuesta resultante determinará el futuro de la organización (Boxall, 2008). Los sindicatos están muy determinados por el entorno y sus cambios pero nunca lo están totalmente y siempre existe un margen de discrecionalidad en las organizaciones sobre la que basar sus apuestas estratégicas de futuro. Dadas las circunstancias del sindicalismo actual, adquirir este enfoque estratégico se convierte en un asunto crítico que requiere de análisis profundos, conocimiento especializado y mucha voluntad de hacer profundos cambios en las organizaciones, desafiando al convervadurismo interno de los aparatos. El enfoque de las fuentes de poder sindical pone el punto de mira en el origen de fuerza de los sindicatos para analizar las causas del debilitamiento y planificar estrategias para el empoderamiento. La ventaja de esta visión es doble ya que, por un lado, contribuye a llevar este análisis al interior de las propias organizaciones sindicales, sin atender a otros factores que, aunque sean importantes, quedan fuera de la capacidad de intervención de los sindicatos. Por otro lado, permite también una lectura estratégica al poner de manifiesto las relaciones que se dan entre las diferentes fuentes de poder sindical. 
Así, se comprueba que los avances y desarrollos en una fuente de poder sindical se extiende fácilmente a otras fuentes, generando una secuencia de empoderamiento. Para ello hay que revertir la tendencia actual en la que el debilitamiento de unas fuentes de poder sindical se extiende hacia las otras en un proceso de retroalimentación con sentido negativo. Como hemos visto, las fuentes de poder sindical de las organizaciones espańolas están en un estado, por lo general, precario y con tendencia hacia un mayor debilitamiento. Es necesario una apuesta transformadora por aumentar el poder sindical que requiere, en primer lugar, comprender la imperiosa necesidad de hacerlo para que las organizaciones sindicales mantengan un papel destacado como actores sociales en representación de los intereses generales de los trabajadores. En segundo lugar, se deben planificar las estrategias minuciosamente para generar un proceso acumulativo de poder sindical a través de diversas fuentes. Finalmente, se debe destinar a esta misión recursos a una escala sin precedentes y vencer muchas resistencias internas y externas para acomenter cambios profundos.

\section{Bibliografía}

Baccaro, Lucio; Hamman, Kerstin y Turner, Lowell (2003): «The Politics of Labour Movement Revitalization: The Need for a Revitalized Perspective», European Journal of Industrial Relations, 9(1), 119-133.

Batstone, Eric (1988): «The frontier of control», en Gallie, Duncan (ed.): Employment in Britain, Oxford, Blackwell, 218-247

Behrens, Martin; Hamman, Kerstin y Hurd, Richard (2004a): "Conceptualizing Labor Union Revitalization» en Frege, Carola y Kelly, John: Varieties of Unionism. Strategies for Union Revitalizacion in a Globalizing Economy, Oxford, Oxford University Press, 11-29.

Behrens, Martin; Hurd, Richard y Waddington, Jeremy (2004b): «How Does Restructuring Contribute to Union Revitalization?», en Carola Frege y John Kelly: Varieties of Unionsm. Strategies for Union Revitalization in a Globalizing Economy, Oxford, Oxford University Press, 117-136.

Boxall, Peter (2008): "Trade Union Strategy», en Blyton, Paul; Heery, Edmund; Bacon Nicolas y Fiorito, Jack The SAGE Handbook of Industrial Relations, Thousand Oaks, SAGE, 209-224.

Bronfenbrenner, Kate; Fiedman, Richard; Hurd, Richard; Oswald, Rudolph y SeeBER, Ronald (1998): Organizing to Win. New Research on Union Strategies. Ithaca, Cornell University Press.

CIS (2015): Barómetro de abril de 2015 del Centro de Investigaciones Sociológicas. Estudio n. ${ }^{\circ}$ 3082. Madrid, CIS.

Eвbinghaus, Bernard (2002): Dinosaurier der Dienstleistungsgesellschaft?, Köhln, Max Planck Institute.

Evans, Peter (2010): «Is it Labor's turn to globalize?. Twenty-first Century Opportunities and Strategic Responses», en IRLE Working Papers, Berkeley, University of California, 352-379.

Frege, Carola y Kelly, John (2004): Varieties of Unionism.Strategies for Union Revitalization in a Globalizing Economy, Oxford, Oxford University Press. 
Gall, Gregor (2008): Sources of Union Power, Hatfied, Centre for Research in Employment Studies.

Gumbrell-McCormick, Richard y Hyman, Richard (2013): Trade Unions in Western Europe. Hard Times, Hard Choices, Oxford, Oxford University Press.

Hamman, Kerstin y Martinez-Lucio, Miguel (2003). Strategies of Union Revitalization in Spain: Negotiating Change and Fragmentation. European Journal of Industrial Relations, 9 (1), 61-78.

Hyman, Richard (1981): Relaciones industriales. Una introducción marxista, Madrid, Blume.

Kloosterboer, Dirk (2007): Innovative Trade Union Strategies. Amsterdam, Stichting FNV Pers.

KöHLER, Holm-Detlev (2008): Los sindicatos en España frente a los retos de la Globalización y el cambio tecnológico, Madrid, Fundación Alternativas.

KöHLER, Holm-Detlev y Calleja, José Pablo (2015) «They don't represent us!» Opportunities for a Social Movement Unionism Strategy in Spain. Relations industrielles/Industrial Relations, 70 (2) 240-261.

LÉvesque, Christian y Murray, Gregor (2010): Understanding union power: resources and capabilities for renewing union capacity. Transfer, 16 (3) 333-350.

McIlroy, John (2000): The new politics of pressure - The Trades Union Congress and new Labour in government. Industrial Relations Journal, 31, 2-16.

Offe, Claus y Helmut Wiesenthal (1980): «Two logics of collective action: Theoretical notes on social class and organizational form», Political Power and Social Theory, 1, $67-115$.

TARrow, Sidney (2004): El poder en movimiento. Los movimientos sociales, la acción colectiva y la politica. Madrid: Alianza.

Tattersall, Amanda (2005): There is Power in Coalition: A framework for assessing how and when union-community coalitions are effective and enhance union power. Labour and Industry, 16 (2), 97-112.

UrbaN, Hans-Jürgen (2012): "Crisis corporatism and Trade Union Revitalisation in Europe» en Steffen Lehndorff, A triumph of failed ideas: European models of capitalism in the crisis, Brussels, ETUI, 219-241. 